\title{
Predicting the size of pulmonary arteriovenous malformations on chest computed tomography: a role for transthoracic contrast echocardiography
}

\author{
Sebastiaan Velthuis ${ }^{1}$, Elisabetta Buscarini², Johannes J. Mager ${ }^{3}$, \\ Veronique M.M. Vorselaars ${ }^{1}$, Marco W.F. van Gent ${ }^{1}$, Pietro Gazzaniga ${ }^{4}$, \\ Guido Manfredi ${ }^{2}$, Cesare Danesino ${ }^{5}$, Arjen L. Diederik ${ }^{6}$, Jan A. Vos ${ }^{6}$, \\ Silvia Gandolfi ${ }^{7}$, Repke J. Snijder ${ }^{3}$, Cornelis J.J. Westermann ${ }^{3}$ \\ and Martijn C. Post ${ }^{1}$
}

Affiliations: 'Dept of Cardiology, St Antonius Hospital, Nieuwegein, ${ }^{3}$ Dept of Pulmonology, St Antonius Hospital, Nieuwegein, and ${ }^{6}$ Dept of Radiology, St Antonius Hospital, Nieuwegein, the Netherlands. ${ }^{2}$ Dept of Gastroenterology, Maggiore Hospital, Crema, ${ }^{4}$ Dept of Cardiology, Maggiore Hospital, Crema, ${ }^{5}$ Dept of Molecular Medicine, University of Pavia and IRCCS, S. Matteo, Pavia, and ${ }^{7}$ Dept of Radiology, Maggiore Hospital, Crema, Italy.

Correspondence: Sebastiaan Velthuis, Dept of Cardiology, St Antonius Hospital, Koekoekslaan 1, 3435 CM, Nieuwegein, the Netherlands. E-mail: S.Velthuisdantoniusziekenhuis.nl

ABSTRACT This study aimed to investigate whether pulmonary shunt grade on transthoracic contrast echocardiography (TTCE) predicts the size of pulmonary arteriovenous malformations (PAVMs) on chest computed tomography (CT) and subsequent feasibility for transcatheter embolotherapy.

We prospectively included 772 persons with possible or definite hereditary haemorrhagic telangiectasia, who underwent both TTCE and chest CT for screening of PAVMs. A quantitative three-point grading scale was used to classify the pulmonary shunt size on TTCE (grade 1-3). Transcatheter embolotherapy was performed for PAVMs deemed large enough for endovascular closure on chest CT.

TTCE documented pulmonary shunting in $510(66.1 \%)$ patients. The positive predictive value of a pulmonary shunt grade 1, 2 and 3 on TTCE for presence of PAVMs on chest CT was $13.4 \%, 45.3 \%$ and $92.5 \%$, respectively $(\mathrm{p}<0.001)$. None of the 201 persons with a pulmonary shunt grade 1 on TTCE had PAVMs on chest CT large enough for transcatheter embolotherapy, while $38(25.3 \%)$ and $123(77.4 \%)$ individuals with a pulmonary shunt grade 2 and 3 on TTCE, respectively, underwent endovascular closure of PAVMs.

Pulmonary shunt grade on TTCE predicts the size of PAVMs on chest CT and their feasibility for subsequent transcatheter embolotherapy. Chest CT can be safely withheld from all persons with a pulmonary shunt grade 1 on TTCE, as any PAVM found in these subjects will be too small for transcatheter embolotherapy.

@ERSpublications

Chest CT can be safely withheld from persons with pulmonary shunt grade 1 on TTCE http://ow.ly/sUAps

Received: Aug 012013 | Accepted after revision: Jan 172014 | First published online: March 062014

Support statement: The study was supported by the Fondazione Italiana HHT “Onilde Carini” (grant 2/2012).

Conflict of interest: None declared.

Copyright @ERS 2014 


\section{Introduction}

Pulmonary arteriovenous malformations (PAVMs) are thin-walled abnormal vessels replacing normal capillaries between the pulmonary arterial and venous circulation, resulting in a permanent right-to-left shunt. The majority of PAVMs $(\sim 90 \%)$ are associated with hereditary haemorrhagic telangiectasia (HHT) [1]. HHT is an autosomal dominant inherited disorder characterised by vascular abnormalities varying from small telangiectasias in the skin and mucous membranes to large arteriovenous malformations, predominantly in the brain, liver and lungs [2]. Most HHT patients have a mutation in the gene coding for endoglin $(H H T 1)$ or activin receptor-like kinase 1 (HHT2) $[3,4]$. A pulmonary shunt has been reported in up to $85 \%$ of HHT1 and in $35 \%$ of HHT2 patients [5]. PAVMs are associated with disabling and lifethreatening complications, such as cerebral ischaemic strokes, brain abscesses, massive haemoptysis or haemothorax $[6,7]$. The neurological complications occur via paradoxical embolisation of thrombotic or septic origin that bypasses the pulmonary capillary filter, whereas the haemorrhagic complications are due to spontaneous rupture of PAVMs. Transcatheter embolotherapy is an endovascular intervention that occludes the feeding artery of the PAVM with a coil or plug, in order to reduce the risk of PAVM-related complications [8]. Because of the high prevalence, potential severe complications and effective treatment options, screening for PAVMs is recommended in all persons with possible or confirmed HHT. During the last few years, transthoracic contrast echocardiography (TTCE) has become the initial screening test for detecting PAVMs, based on its excellent sensitivity, negative predictive value, good inter-observer reproducibility and noninvasive character, with low risks and costs [5, 9-11]. TTCE can be used to estimate the pulmonary shunt size, varying from small (grade 1), moderate (grade 2), to large (grade 3) right-to-left shunts (fig. 1). Irrespective of this echocardiographic pulmonary shunt grade, the current international guideline for diagnosis and management of HHT and PAVMs requires the confirmation of all pulmonary shunts on TTCE by chest computed tomography (CT) with thin-cut reconstructions, in order to evaluate the necessity for transcatheter embolotherapy [9]. However, it remains unclear whether additional chest CT is indeed mandatory in all persons with only a small pulmonary shunt on TTCE, as these shunts may be too small for subsequent transcatheter embolotherapy [12-14]. Therefore, this large, prospective two-centre study investigated whether pulmonary shunt grade on TTCE predicts the size of PAVMs on chest CT and subsequent feasibility for transcatheter embolotherapy.

\section{Methods \\ Study population}

From May 2004 to October 2012, 1280 consecutive individuals were screened for HHT at two specialised clinics: the St Antonius Hospital in Nieuwegein (the Netherlands) and the Maggiore Hospital in Crema (Italy). Persons aged $>15$ years were screened if they were family members of index patients with HHT or had clinical symptoms suggesting HHT. TTCE was performed in 1240 (96.9\%) individuals; placement of an intravenous line failed or was refused in the other $40(3.1 \%)$ persons. In $50(4.0 \%)$ out of these 1240 persons, no clear distinction was possible between a pulmonary or cardiac right-to-left shunt and these "indeterminate" shunts were excluded from further analysis. Chest CT was performed in 1020 out of the remaining $1190(85.7 \%)$ individuals, as chest CT was withheld in patients without a pulmonary shunt on TTCE from January 2011. All 1020 persons underwent complete history and physical examination by a physician with dedicated expertise in HHT. The clinical diagnosis of HHT was established according to the Curaçao criteria [15]. These criteria consist of spontaneous and recurrent epistaxis, telangiectasias at characteristic sites, visceral arteriovenous malformations, and a first-degree relative with HHT. Genetic testing for the HHT-causing gene mutation was offered to all subjects and performed as described previously $[16,17]$. A definite diagnosis of HHT was established in case of three or more clinical Curaçao criteria, or when genetic testing identified the HHT-causing mutation. The diagnosis of HHT was "possible" in patients with two clinical Curaçao criteria without genetic testing or if a mutation could not be found without knowing a specific family mutation. The diagnosis of HHT was "unlikely" in the presence of less than two clinical Curaçao criteria, without genetic testing or if a mutation could not be found without knowing a specific family mutation. A diagnosis of HHT was rejected when genetic testing excluded the known HHT-causing family mutation. Because the pre-test probability of having PAVMs is smaller in persons without HHT, we excluded all persons where HHT was rejected $(n=157)$ or unlikely $(n=91)$. Therefore, a total of 772 patients with possible or definite HHT in which both TTCE and chest CT were performed were included in the statistical analyses of the present study (fig. 2). Patients with previously treated PAVMs were not included in the analysis. The study was approved by the institutional review boards of both hospitals (17/2004 (St Antonius Hospital) and LTME/Z-12.41 (Maggiore Hospital)).

\section{Transthoracic contrast echocardiography}

TTCE was performed by placing an intravenous line in the right antecubital vein to which two $10-\mathrm{mL}$ syringes were connected, one filled with $8 \mathrm{~mL}$ physiological saline solution and the other with $1 \mathrm{~mL}$ air. 

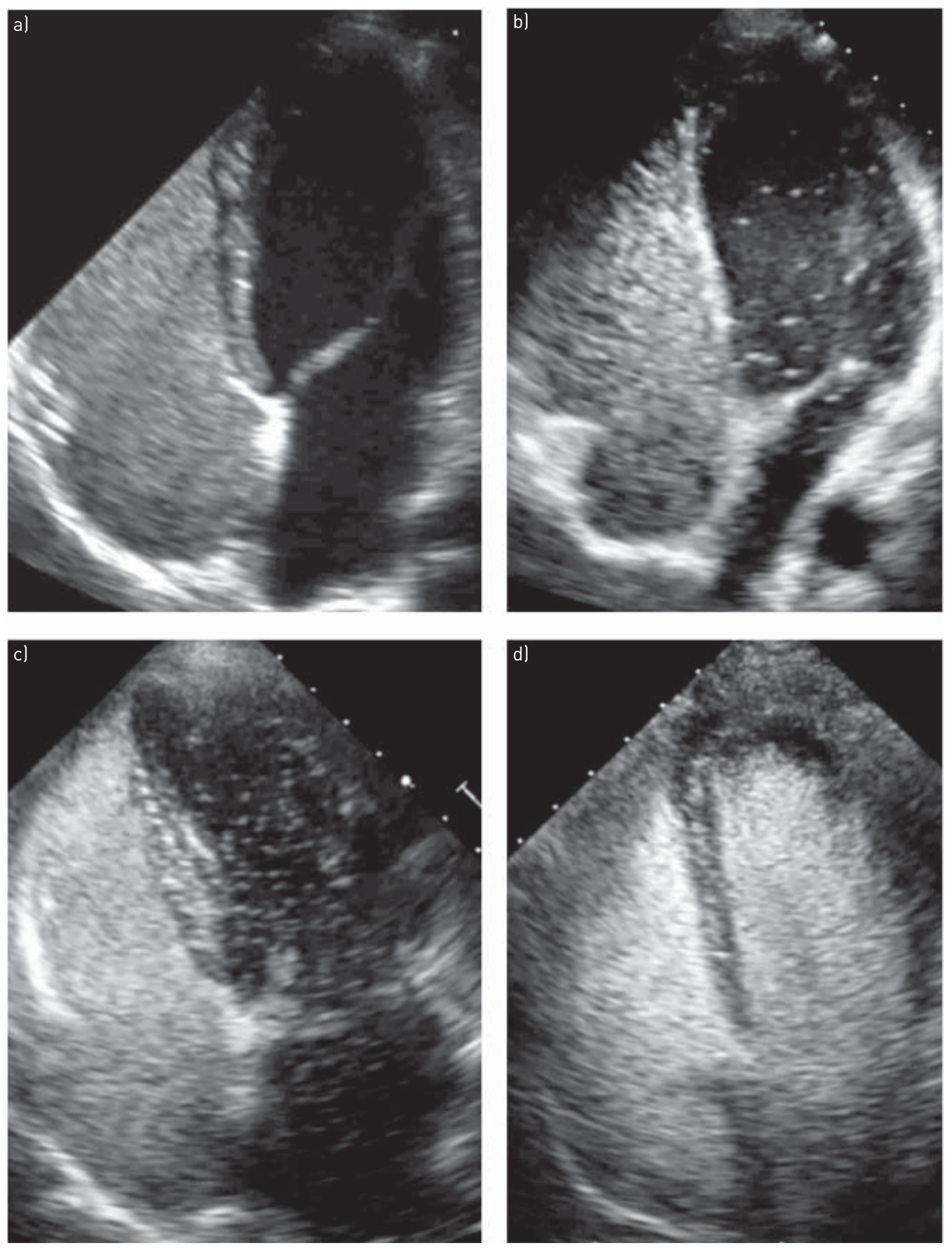

FIGURE 1 Apical four-chamber view using transthoracic contrast echocardiography demonstrating a) no right-to-left shunt, b) pulmonary shunt grade 1, c) pulmonary shunt grade 2 and d) pulmonary shunt grade 3 .

Subsequently, $1 \mathrm{~mL}$ blood was drawn into the air-filled syringe and mixed with the saline by reverse flushing between both syringes, creating agitated saline with microbubbles. The patient was positioned in the left lateral position and $5 \mathrm{~mL}$ agitated saline was injected within $3 \mathrm{~s}$ while projecting the four-chamber apical view, with and without a Valsalva manoeuvre. TTCE was performed by a constant group of three trained echocardiographers. Shunt interpretation was performed by two experienced cardiologists with dedicated expertise in HHT who were unaware of the patient's medical history. In the case of a right-to-left shunt, visualisation of shunt origin was pursued in every TTCE. All right-to-left shunts visualised through a pulmonary vein were classified as pulmonary shunts. In the case of poor visualisation of shunt origin, we used a delay of four cardiac cycles to distinguish a pulmonary from a cardiac right-to-left shunt, in which TTCE was considered positive for a pulmonary shunt if microbubbles appeared in the left atrium after four 
FIGURE 2 Selection of study population. HHT: hereditary haemorrhagic telangiectasia; RLS: right-to-left shunt; TTCE: transthoracic contrast echocardiography; CT: computed tomography. "* additional chest CT was withheld in the absence of a pulmonary shunt on TTCE from January 2011.

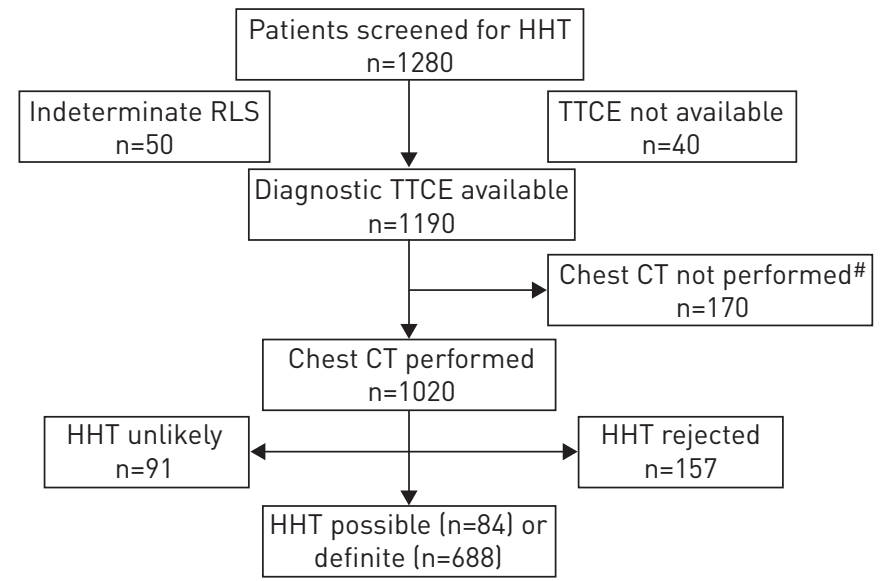

or more cardiac cycles, as described previously $[5,11,14]$. Based on the maximum number of microbubbles counted in the left side of the heart in one still frame, the shunt was graded as 1 (maximum of 29 microbubbles), 2 (30-100 microbubbles) or 3 (>100 microbubbles), as reported previously $[10,11,18]$. In the case of disagreement on presence, quantity or timing of microbubbles in the left ventricle, the TTCE was reviewed again by both cardiologists together to agree on the final determination.

\section{Chest CT}

Chest CT was routinely performed with a $\geqslant 16$-detector CT scanner (Philips Medical Systems, Amsterdam, the Netherlands) with a dedicated high-resolution algorithm and maximum slice thickness of $1 \mathrm{~mm}$ in both hospitals. Identification of PAVMs on chest CT was based on the presence of a nodular opacity with both an afferent and efferent vessel. Chest CT images were evaluated in a multidisciplinary consensus meeting with dedicated interventional radiologists and vascular pulmonologists, who were blinded to the results of TTCE. When observers disagreed, chest CT was considered positive for a PAVM and additional pulmonary angiography of the pulmonary artery was performed, given the impact of complications from a potentially missed treatable PAVM.

\section{Transcatheter embolotherapy of PAVMs}

Based on the collective experience of the present HHT community, all PAVMs with a feeding artery diameter of $\geqslant 3 \mathrm{~mm}$ were deemed accessible to transcatheter embolotherapy, whereas diameters $<2 \mathrm{~mm}$ were considered to indicate inaccessibility for endovascular closure. Feasibility of transcatheter embolotherapy of PAVMs with a feeding artery diameter between 2 and $3 \mathrm{~mm}$ was based on technical aspects of the endovascular intervention, which was also assessed in the aforementioned multidisciplinary consensus meeting. Features taken into account in these cases included location, tortuosity, angulation and multiplicity of PAVMs. The decision to treat PAVMs was blinded to the results of TTCE and potential clinical significance of the PAVM (for example, a prior cerebral paradoxical complication), as treatment decisions could otherwise have been influenced, which was not the case in the present study. The feeding artery diameters of embolised PAVMs on both chest CT and pulmonary angiography (based on the calibre of the embolisation catheter) were measured in order to analyse their degree of agreement. Transcatheter embolotherapy of PAVMs was performed by a constant group of three and two interventional radiologists in Nieuwegein and Crema, respectively, using platinum or stainless steel coils, endovascular plugs, or a combination of these materials.

\section{Statistical analysis}

Descriptive statistics were used to describe the patient characteristics. The positive predictive value (PPV) of the different pulmonary shunt grades on TTCE was calculated using chest CT as a reference. The MantelHaenszel Chi-squared test was used to determine whether there was a significant association between the pulmonary shunt grade on TTCE and the presence of PAVMs on chest CT. A Bland-Altman analysis was performed to evaluate the degree of agreement in PAVM feeding artery diameter on chest CT and pulmonary angiography. Statistical analyses were performed using the statistical software application SPSS (version 17.0; SPSS Inc., Chicago, IL, USA). 
TABLE 1 Overall patient characteristics by pulmonary shunt grade on transthoracic contrast echocardiography

\begin{tabular}{|c|c|c|c|c|c|}
\hline & No shunt & Grade 1 & Grade 2 & Grade 3 & Total \\
\hline Persons & 262 (33.9) & $201(26.1)$ & $150(19.4)$ & $159(20.6)$ & 772 \\
\hline Age years mean $\pm S D$ & $47.3 \pm 15.1$ & $44.9 \pm 16.1$ & $43.7 \pm 16.0$ & $44.8 \pm 14.2$ & $45.5 \pm 15.4$ \\
\hline \multicolumn{6}{|l|}{ Sex } \\
\hline Females & $146(32.5)$ & $113(25.2)$ & $79(17.6)$ & $111(24.7)$ & 449 (58.2) \\
\hline Males & $116(35.9)$ & $88(27.2)$ & $71(22.0)$ & $48(14.9)$ & 323 (41.8) \\
\hline \multicolumn{6}{|l|}{ HНT } \\
\hline \multicolumn{6}{|l|}{ Genetic testing } \\
\hline HHT1 & $21(9.4)$ & $39(17.4)$ & $57(25.4)$ & 107 (47.8) & $224(29.0)$ \\
\hline HНT2 & $145(47.5)$ & $104(34.1)$ & 38 (12.5) & $18(5.9)$ & 305 (39.5) \\
\hline SMAD4 & $1(10.0)$ & $4(40.0)$ & $2(20.0)$ & $3(30.0)$ & 10 (1.3) \\
\hline Unknown ${ }^{\#}$ & 95 (40.8) & $54(23.2)$ & $53(22.7)$ & 31 (13.3) & $233(30.2)$ \\
\hline
\end{tabular}

Data are presented as $\mathrm{n}(\%)$, unless otherwise stated. HHT: hereditary haemorrhagic telangiectasia. ${ }^{\#}$ : unknown family mutation or genetic testing not performed.

\section{Results}

\section{Study population}

A total of 772 patients with possible or definite HHT (mean age $45.5 \pm 15.4$ years, $58.2 \%$ females) underwent both TTCE with pulmonary shunt grade measurement and chest CT for the detection of PAVMs (497 at St Antonius Hospital and 275 at Maggiore Hospital). The patient characteristics and homogeneity between these centres are described in tables 1 and 2. Notably, a proportion of these HHT patients has been described before $[10,11]$. The presence of HHT was definite in $688(89.1 \%)$ patients and remained possible in $84(10.9 \%)$ persons. Genetic testing identified $224(29.0 \%)$ patients with HHT1, $305(39.5 \%)$ patients

\section{TABLE 2 Homogeneity of study population}

\begin{tabular}{|c|c|c|}
\hline & $\begin{array}{l}\text { St Antonius Hospital, } \\
\text { Nieuwegein, the } \\
\text { Netherlands }\end{array}$ & $\begin{array}{l}\text { Maggiore Hospital, } \\
\text { Crema, Italy }\end{array}$ \\
\hline Persons & $497(64.4)$ & $275(35.6)$ \\
\hline Age years mean $\pm S D$ & $44.8 \pm 15.5$ & $46.6 \pm 15.2$ \\
\hline \multicolumn{3}{|l|}{ Sex } \\
\hline Females & $296(59.6)$ & $153(55.6)$ \\
\hline Males & $201(40.4)$ & $122(44.4)$ \\
\hline \multicolumn{3}{|l|}{ HHT } \\
\hline Definite & $428(86.1)$ & 260 (94.5) \\
\hline Possible & $69(13.9)$ & 15 (5.5) \\
\hline \multicolumn{3}{|l|}{ Genetic testing } \\
\hline HHT1 & $177(35.6)$ & $47(17.1)$ \\
\hline HHT2 & 208 (41.9) & 97 (35.3) \\
\hline SMAD4 & $9(1.8)$ & $1(0.4)$ \\
\hline Unknown $n^{\#}$ & $103(20.7)$ & $130(47.2)$ \\
\hline \multicolumn{3}{|l|}{ Pulmonary shunt on TTCE } \\
\hline No shunt & $196(39.4)$ & $66(24.0)$ \\
\hline Grade 1 & $109(21.9)$ & $92(33.5)$ \\
\hline Grade 2 & 76 (15.3) & $74(26.9)$ \\
\hline Grade 3 & $116(23.4)$ & $43(15.6)$ \\
\hline PAVM on chest $\mathrm{CT}$ & $158(31.8)$ & 90 (32.7) \\
\hline Transcatheter embolotherapy performed n/N (\%) & $92 / 158(58.2)$ & $69 / 90(76.7)$ \\
\hline
\end{tabular}


with HHT2 and $10(1.3 \%)$ patients with a SMAD4 mutation. DNA analysis could not detect an HHTcausing mutation or was not performed in the other $233(30.2 \%)$ patients.

\section{TTCE and chest CT}

TTCE documented a pulmonary shunt of grade 1 in 201 (26.1\%) patients, grade 2 in $150(19.4 \%)$ patients and grade 3 in $159(20.6 \%)$ patients. We found a good $\kappa$-coefficient of 0.85 for interobserver agreement concerning the pulmonary shunt grade on TTCE, which is in line with prior reports $[5,10,19]$. Chest CT confirmed the presence of a PAVM in $242(47.5 \%)$ out of 510 patients with a pulmonary shunt on TTCE. The remaining $268(52.5 \%)$ patients represented diffuse or microscopic pulmonary shunts below the detection limit of chest CT. In total, 203 (90.6\%) out of 224 HHT1 patients had a pulmonary shunt on TTCE and $137(61.2 \%)$ of them also had a PAVM on chest CT. A pulmonary shunt on TTCE was documented in $160(52.5 \%)$ out of 305 HHT2 patients and $42(13.8 \%)$ of them had a PAVM on chest CT.

The PPV of the different pulmonary shunt grades on TTCE for the presence of PAVMs on chest CT was 13.4\% (95\% CI 9.4-18.8), 45.3\% (95\% CI 37.6-53.3) and 92.5\% (95\% CI 87.3-95.6) for pulmonary shunt grade 1,2 and 3 , respectively $(\mathrm{p}<0.001)$ (fig. 3). Six $(0.8 \%)$ out of 772 patients had a PAVM-like structure on chest CT without a pulmonary shunt on TTCE. This could not be explained by poor quality of TTCE or chest CT, or disagreement between interpreters. These sporadic cases were considered as false-positive chest CT and antibiotic prophylaxis was not advised. In $22(8.4 \%)$ out of 262 patients without a pulmonary shunt on TTCE, there was evidence of a patent foramen ovale. None of these persons was diagnosed with PAVMs on chest CT.

a)

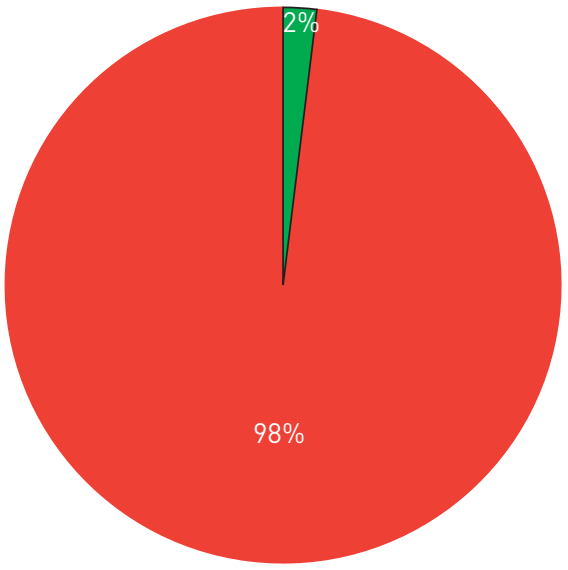

c)

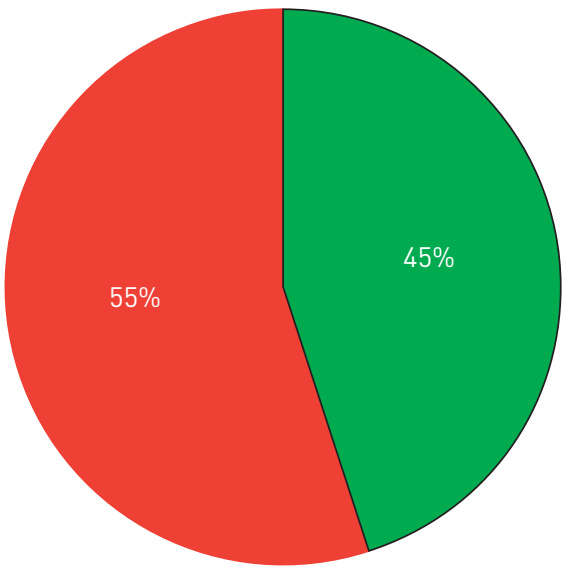

PAVM present on chest CT

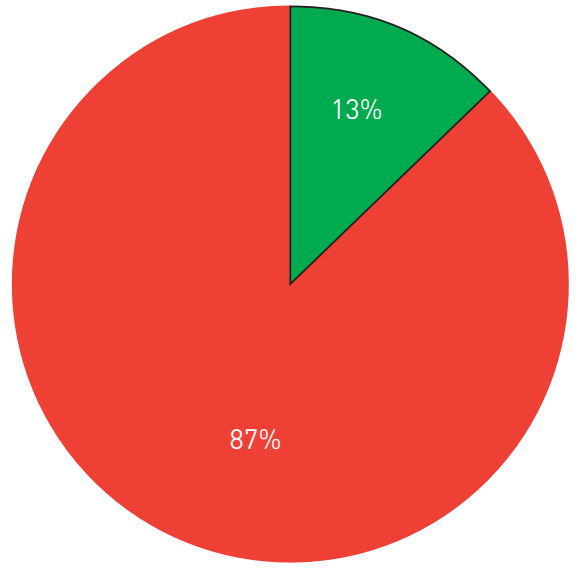

d)

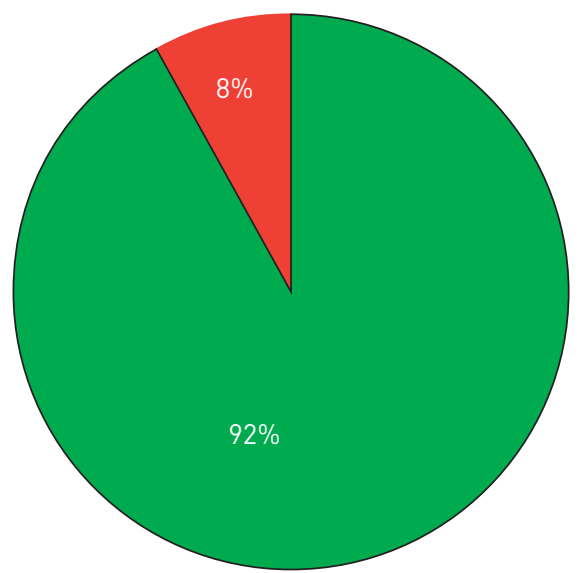

No PAVM on chest CT

FIGURE 3 Pulmonary shunt grade on transthoracic contrast echocardiography (TTCE) predicts presence of pulmonary arteriovenous malformations (PAVMs) on chest computed tomography (CT). a) No shunt $(n=262)$; b) grade $1(n=201)$; c) grade $2(\mathrm{n}=150)$; d) grade $3(\mathrm{n}=159)$. 


\section{Transcatheter embolotherapy of PAVMs}

Transcatheter embolotherapy of PAVMs was performed in 161 (31.6\%) out of 510 patients with a pulmonary shunt on TTCE. Most patients had a PAVM feeding artery diameter on chest CT of $\geqslant 3 \mathrm{~mm}$ (88\%), while only $12 \%$ had a feeding artery diameter between 2.0 and $2.9 \mathrm{~mm}$ (table 3). Our Bland-Altman analysis (fig. 4) demonstrated that chest CT accurately estimated the PAVM feeding artery diameter on pulmonary angiography, with a mean difference of $-0.03 \mathrm{~mm}(95 \% \mathrm{CI}-1.12-1.06 \mathrm{~mm})$ between these techniques and concordance correlation coefficient of 0.94 . The majority of transcatheter embolotherapy was performed in patients with HHT1 (59.0\% HHT1, 13.7\% HHT2, 1.2\% SMAD4 and 26.1\% unknown mutation or DNA analysis not performed). In total, 95 (42.4\%) out of 224 HHT1 patients underwent transcatheter embolotherapy versus only $22(7.2 \%)$ out of 305 HHT2 patients. The necessity of endovascular closure of PAVMs was strongly related with pulmonary shunt grade on TTCE (table 4). The PPV of pulmonary shunt grade 1, 2 and 3 on TTCE for the feasibility of transcatheter embolotherapy of PAVMs was $0 \%, 25.3 \%$ (95\% CI 19.1-32.9\%) and 77.4\% (95\% CI 70.3-83.2\%), respectively (table 4 and fig. 5). Based on chest CT, none of the 262 and 201 patients with no or grade 1 pulmonary shunt on TTCE, respectively, had PAVMs large enough for subsequent transcatheter embolotherapy, whereas endovascular closure was performed in 38 out of 150 and 123 out of 159 patients with pulmonary shunt grade 2 and 3, respectively, on TTCE.

\section{Discussion}

The present large prospective study demonstrates that pulmonary shunt grade on TTCE not only correlates with the probability of detecting PAVMs on chest CT but also, and most importantly, predicts the feasibility of subsequent transcatheter embolotherapy. The main strength of our study is the finding that persons with pulmonary shunt grade 1 on TTCE do not have PAVMs large enough for transcatheter embolotherapy. Therefore, additional chest CT can be safely withheld from all these individuals, which may improve the current management strategy of pulmonary right-to-left shunts.

The present guideline for the diagnosis and treatment of HHT recommends TTCE as the initial screening test for pulmonary shunts [9] in order to detect PAVMs before the development of potential severe complications. Some centres advocate performing chest radiography before TTCE in order to rule out large PAVMs, where TTCE might be withheld. With a low sensitivity of only $28 \%$ compared with chest CT [11], we believe that chest radiography lacks diagnostic value as screening test for PAVMs as large pulmonary shunts will still be missed in the majority of patients and unnecessary radiation exposure can be prevented. Furthermore, the safety of TTCE has been well-documented [20], which is supported by a very low incidence of minimal and self-resolving side-effects in the recent literature $[10,13]$.

In the last few years, a quantitative three-point grading scale was developed to classify pulmonary shunt size on TTCE, differentiating between grade 1, 2 and 3 (small, moderate or large, respectively) [14]. Our present study confirms that an increased pulmonary shunt grade on TTCE correlates with an increased probability of detecting PAVMs on chest CT [13]. Still, chest CT remains negative in $54.7 \%$ and $7.5 \%$ of patients with pulmonary shunt grade 2 and 3 on TTCE, respectively. These shunts represent diffuse microscopic PAVMs below the detection limit of chest CT. This underscores the importance of TTCE, as these moderate to large

TABLE 3 Distribution of pulmonary arteriovenous malformation (PAVM) feeding artery diameter on chest computed tomography (CT) of embolised PAVMs

PAVM feeding artery diameter on chest CT $\mathrm{mm}$

Patients n (\%)

\begin{tabular}{lc}
\hline $\mathbf{2 . 0}-\mathbf{2 . 9}$ & $18(12.0)$ \\
$\mathbf{3} . \mathbf{-}-\mathbf{9} .9$ & $51(34.0)$ \\
$\mathbf{4 . 0}-\mathbf{4 . 9}$ & $40(26.7)$ \\
$\mathbf{5 . 0}-\mathbf{5 . 9}$ & $17(11.3)$ \\
$\mathbf{6 . 0}-\mathbf{6 . 9}$ & $10(6.7)$ \\
$\mathbf{7 . 0 - 7 . 9}$ & $7(4.7)$ \\
$\mathbf{8 . 0 - 8 . 9}$ & $4(2.6)$ \\
$\mathbf{9 . 0 - 9 . 9}$ & $1(0.7)$ \\
$>10.0$ & $2(1.3)$ \\
\hline
\end{tabular}

Measurement of PAVM feeding artery diameters on chest CT was available in 150 (93.2\%) out of 161 embolised patients. Largest diameter used in case of multiple PAVMs or feeders in one patient. 
FIGURE 4 Bland-Altman analysis demonstrating good degree of agreement in pulmonary arteriovenous malformation feeding artery diameter between chest computed tomography (CT) and pulmonary angiography (angio).

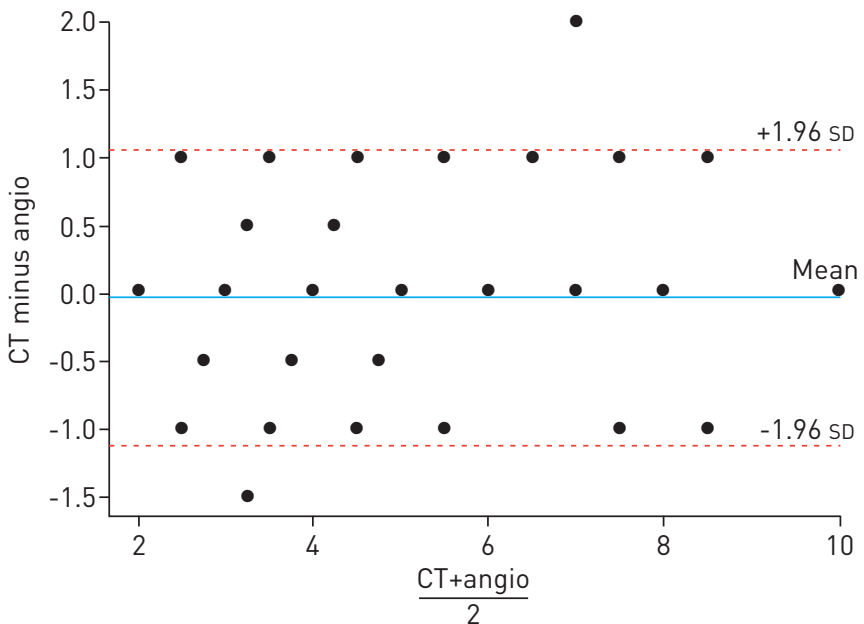

pulmonary shunts are missed with chest CT, but do carry an increased risk of paradoxical cerebral complications and deserve antibiotic prophylaxis for procedures with a high risk of bacteraemia [18].

The present international guideline on diagnosis and management of HHT recommends confirmation of every pulmonary shunt on TTCE by chest CT, in order to evaluate the opportunity for transcatheter embolotherapy [9]. Currently, selection of PAVMs suitable for transcatheter embolotherapy is based on the PAVM feeding artery diameter on chest CT, generally $\geqslant 3 \mathrm{~mm}$, although targeting PAVMs with a feeding artery diameter as low as $2 \mathrm{~mm}$ may be appropriate [9]. It has been hypothesised that additional chest CT might be withheld from persons with only a small pulmonary shunt on initial TTCE because these PAVMs might be too small for endovascular closure, but large studies are lacking [12, 14]. Given the impact of a potential complication from a missed treatable PAVM, more data were required before re-evaluating the necessity of additional chest CT in persons with only a small pulmonary shunt on TTCE. The present large prospective two-centre study now demonstrates that only $13 \%$ of patients with a pulmonary shunt grade 1 on TTCE have detectable PAVMs on chest CT and none of these PAVMs is large enough for transcatheter embolotherapy. Therefore, TTCE may predict the feasibility of transcatheter embolotherapy and discern clinically insignificant from treatable PAVMs. Interestingly, our group recently reported that pulmonary shunt grade on TTCE is also associated with the prevalence of neurological complications in patients screened for HHT [18]. In that large cross-sectional study [18], a pulmonary shunt grade 1 on TTCE did not appear to be a risk factor for the presence of neurological complications. Together with the findings of the present study, these data contribute important new elements to the challenging discussion about the optimal approach to persons with only a small pulmonary shunt on initial TTCE. In the present study, a pulmonary shunt grade 1 on TTCE was present in $26.0 \%$ of patients with possible or definite HHT, where additional chest CT could have been withheld. This percentage can even be higher in a general screening population for HHT, as small pulmonary shunts are also described in $6-28 \%$ of healthy subjects $[5,10,21]$. Deferring additional chest CT in all persons with pulmonary shunt grade 1 on TTCE could result in a significant cost saving and reduction of radiation exposure in mainly young adults [12]. Using this strategy,

TABLE 4 Number of patients who underwent transcatheter embolotherapy of pulmonary arteriovenous malformations (PAVMs) by pulmonary shunt grade on transthoracic contrast echocardiography (TTCE)

Pulmonary shunt on TTCE

Persons $\mathbf{n}$

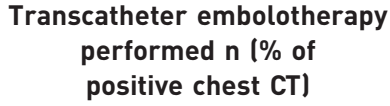

PPV \% $[95 \%$ Cl] n $(\%)$

$0(0)$
$0(0)$
$38(55.9)$
$123(83.7)$
$161(64.9)$

$77.4(70.3-83.2)$

$\begin{array}{lll}\text { Grade } 3 & 159 & 147(92.5) \\ \text { Total } & 772 & 248(32.1)\end{array}$

CT: computed tomography; PPV: positive predictive value. 
a)

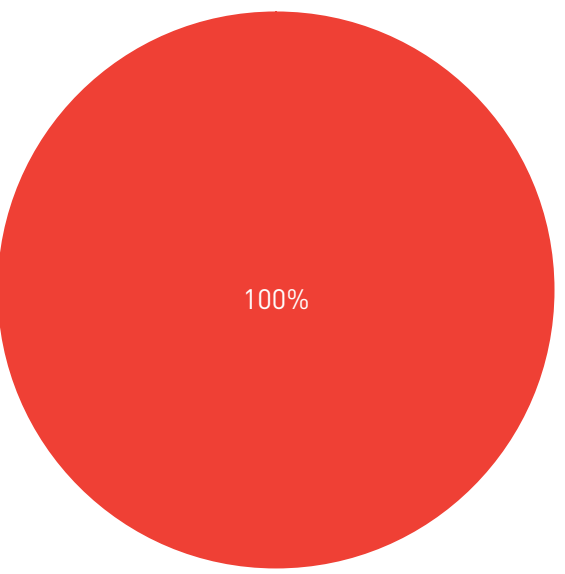

c)

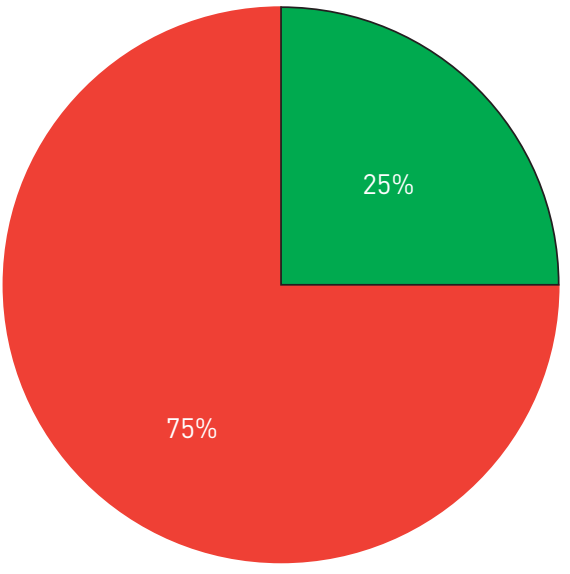

Embolotherapy performed b)

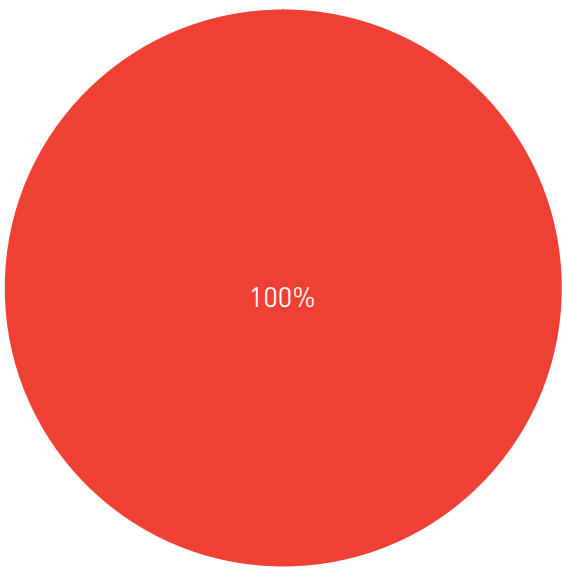

d)

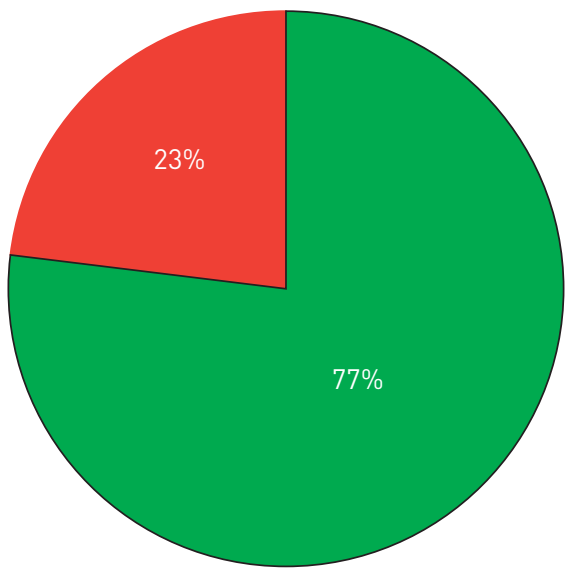

Embolotherapy not performed

FIGURE 5 Pulmonary shunt grade on transthoracic contrast echocardiography predicts feasibility for transcatheter embolotherapy of pulmonary arteriovenous malformations on chest computed tomography. a) No shunt $(n=262)$; b) grade $1(\mathrm{n}=201) ; \mathrm{c})$ grade $2(\mathrm{n}=150)$; $)$ grade $3(\mathrm{n}=159)$.

additional chest CT would have been indicated in only $40 \%$ of our study population with possible or definite HHT.

\section{Study limitations}

First, our study population consisted of HHT patients screened at two hospitals with specific experience in pulmonary shunt grading with TTCE and it remains uncertain whether our results also apply to a general population in centres without this expertise. However, screening for PAVMs with TTCE is preferably performed in specialised HHT centres in order to achieve the accuracy reported in the literature. Second, it can be challenging to discern an early pulmonary shunt from an intracardiac patent foramen ovale using TTCE. In the present study, no clear distinction between shunt origin was possible in 50 (4.0\%) out of 1040 initially screened persons, who were excluded from further analysis. In this small subset of individuals with an indeterminate right-to-left shunt, the diagnostic accuracy of TTCE is limited and additional chest CT remains indicated for the detection of potential treatable PAVMs. Third, our study did not record detailed echocardiographic data on left ventricular function, valvular heart disease, right ventricular systolic pressure, heart rate, cardiac output or anaemia, which may have influenced the delay and size of contrast appearance in the left ventricle. Fourth, not all patients in our study underwent a pulmonary angiography to confirm the results of chest CT, as this is unethical and not advised in the current guideline. Chest CT is therefore essentially elevated to being the gold standard for determining the feasibility for transcatheter embolotherapy of PAVMs. Although our Bland-Altman analysis indicates that chest CT accurately estimates the feeding artery diameter of large PAVMs on pulmonary angiography, this remains unknown for smaller PAVMs (where pulmonary angiography was not performed). Fifth, feasibility of transcatheter embolotherapy of PAVMs with a feeding artery diameter $<3 \mathrm{~mm}$ was based on technical aspects of the 
endovascular intervention, but in daily practice, this decision will at least partly remain subject to personal judgment as international accepted treatment criteria are currently lacking. Furthermore, treatment decisions may be influenced by pulmonary shunt grade on TTCE and potential clinical significance of the PAVM, which was not the case in the present study. These decisions to treat PAVMs are preferentially based on consensus meetings in centres with specific expertise in this endovascular intervention. Finally, we do not yet have sufficient long-term data on potential growth of PAVMs, but it seems conceivable that followup of patients with a pulmonary shunt grade 1 on initial TTCE might be performed by TTCE every 5 years. Chest CT should then only be performed if the echocardiographic pulmonary shunt size increases.

\section{Conclusions}

Pulmonary shunt grade on TTCE not only correlates with the probability of detecting PAVMs on chest CT but also, and most importantly, predicts the feasibility for subsequent transcatheter embolotherapy. Additional chest CT can be safely withheld from all persons with a pulmonary shunt grade 1 on TTCE, as any PAVM found in these subjects will be too small for transcatheter embolotherapy.

\section{Acknowledgements}

We acknowledge the collaboration of C. Olivieri, C. Canzonieri and F. Ornati (Dept of Molecular Medicine, University of Pavia, Pavia, Italy) for the genetic testing in Italian patients. We thank J.C. Kelder (Dept of Cardiology, St Antonius Hospital, Nieuwegein, the Netherlands) for his statistical support.

\section{References}

Cartin-Ceba R, Swanson KL, Krowka MJ. Pulmonary arteriovenous malformations. Chest 2013; 144: 1033-1044. Guttmacher AE, Marchuk DA, White RIJ. Hereditary hemorrhagic telangiectasia. N Engl J Med 1995; 333: 918-924. Berg JN, Gallione CJ, Stenzel TT, et al. The activin receptor-like kinase 1 gene: genomic structure and mutations in hereditary hemorrhagic telangiectasia type 2. Am J Hum Genet 1997; 61: 60-67.

4 McAllister KA, Grogg KM, Johnson DW, et al. Endoglin, a TGF- $\beta$ binding protein of endothelial cells, is the gene for hereditary haemorrhagic telangiectasia type 1. Nat Genet 1994; 8: 345-351.

5 van Gent MW, Post MC, Snijder RJ, et al. Real prevalence of pulmonary right-to-left shunt according to genotype in patients with hereditary hemorrhagic telangiectasia: a transthoracic contrast echocardiography study. Chest 2010; 138: 833-839.

6 Cottin V, Plauchu H, Bayle JY, et al. Pulmonary arteriovenous malformations in patients with hereditary hemorrhagic telangiectasia. Am J Respir Crit Care Med 2004; 169: 994-1000.

7 Gossage JR, Kanj G. Pulmonary arteriovenous malformations. A state of the art review. Am J Respir Crit Care Med 1998; 158: 643-661.

8 Faughnan ME, Lui YW, Wirth JA, et al. Diffuse pulmonary arteriovenous malformations: characteristics and prognosis. Chest 2000; 117: 31-38.

9 Faughnan ME, Palda VA, Garcia-Tsao G, et al. International guidelines for the diagnosis and management of hereditary haemorrhagic telangiectasia. J Med Genet 2011; 48: 73-87.

10 Gazzaniga P, Buscarini E, Leandro G, et al. Contrast echocardiography for pulmonary arteriovenous malformations screening: does any bubble matter? Eur J Echocardiogr 2009; 10: 513-518.

11 van Gent MW, Post MC, Luermans JG, et al. Screening for pulmonary arteriovenous malformations using transthoracic contrast echocardiography: a prospective study. Eur Respir J 2009; 33: 85-91.

12 Gossage JR. Role of contrast echocardiography in screening for pulmonary arteriovenous malformation in patients with hereditary hemorrhagic telangiectasia. Chest 2010; 138: 769-771.

13 Parra JA, Bueno J, Zarauza J, et al. Graded contrast echocardiography in pulmonary arteriovenous malformations. Eur Respir J 2010; 35: 1279-1285.

14 van Gent MW, Post MC, Snijder RJ, et al. Grading of pulmonary right-to-left shunt with transthoracic contrast echocardiography: does it predict the indication for embolotherapy? Chest 2009; 135: 1288-1292.

15 Shovlin CL, Guttmacher AE, Buscarini E, et al. Diagnostic criteria for hereditary hemorrhagic telangiectasia (Rendu-Osler-Weber syndrome). Am J Med Genet 2000; 91: 66-67.

16 Letteboer TG, Zewald RA, Kamping EJ, et al. Hereditary hemorrhagic telangiectasia: ENG and ALK-1 mutations in Dutch patients. Hum Genet 2005; 116: 8-16.

17 Olivieri C, Pagella F, Semino L, et al. Analysis of ENG and ACVRL1 genes in 137 HHT Italian families identifies 76 different mutations (24 novel). Comparison with other European studies. J Hum Genet 2007; 52: 820-829.

18 Velthuis S, Buscarini E, van Gent MW, et al. Grade of pulmonary right-to-left shunt on contrast echocardiography and cerebral complications: a striking association. Chest 2013; 144: 542-548.

19 Zukotynski K, Chan RP, Chow CM, et al. Contrast echocardiography grading predicts pulmonary arteriovenous malformations on CT. Chest 2007; 132: 18-23.

20 Bommer WJ, Shah PM, Allen H, et al. The safety of contrast echocardiography: report of the Committee on Contrast Echocardiography for the American Society of Echocardiography. J Am Coll Cardiol 1984; 3: 6-13.

21 Woods TD, Harmann L, Purath T, Ramamurthy S, et al. Small- and moderate-size right-to-left shunts identified by saline contrast echocardiography are normal and unrelated to migraine headache. Chest 2010; 138: 264-269. 